For Physica Scripta, Proc. of 2nd Int'l Workshop on Auger Spectroscopy and and Electronic Structure

\title{
AUGER SPECTROSCOPY AND ELECTRONICALLY-STIMULATED SURFACE PROCESSES
}

SAND--91-1978C

D. R. Jennison, A. R. Burns, and E. B. Stechel DE92 000950
Condensed Matter Theory Division 1151

Sandia National Laboratories, Albuquerque, NY 87185, US OCT 211991

\begin{abstract}
Electronic excitations in adsorbate layers stimulate desorption and dissociation of adsorbed molecules as well as chemical reactions between adsorbates. The highest-probability stimulated processes produce neutral desorbates and determine how surface composition is altered by electron or photon radiation. A basic understanding has emerged, due largely to laser resonance-enhanced multi-photon ionization (REMPI) experiments, which provide quantum-state resolution of the gas-phase products.

Auger phenomena enter this understanding in several ways. For example, CV Auger spectroscopy determines the screened hole-hole interaction, $U$, in adsorbates, which in turn provides insight into the degree of charge-transfer screening from the substrate. In those systems where screening charge is used in excitation Auger decay, screening directly determines the lifetime, which in turn can exponentially affect the yield. Reductions in screening, e.g. induced by coadsorption of electro-negative species, thus can result in giant yield enhancements. As separate issues, a finite $U$ may prevent the fast resonant decay and thus increase the yield from two-hole excitations, as has been suggested for $\mathrm{NO}_{2}$ dissociation on $\mathrm{Pt}(111)$, or may assist in the localization (self-trapping) of two-hole excitations in dense adsorbate layers, as apparently is the case for NO desorption from the same surface. The latter causes the yields from one- and two-hole excitations to differ in their coverage dependence. Finally, CVV Auger spectroscopy, of course, measures the energies of two-hole excitations, which can be correlated with observed stimulated thresholds.
\end{abstract}

\section{MASTER}

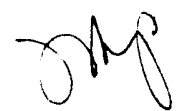

OISTRIBUTION OF T! IIS DOCUMENT IS UNLIMITED 


\section{INTRODUCTION}

Electronic excitations, produced by photon, electron, or ion impact, cause the desorption of atoms and molecules from surfaces. Desorbates may be adsorbates, substrate atoms, or the products of stimulated chemical reactions or adsorbate dissociation. These processes come under the general heading of Desorption Induced by Electronic Transitions (or "DIET"), the subject of a series of workshops [1]. When stimulated reaction or dissociation products directly-desorb, their study offers a uniquely detailed view into the dynamics of condensed phase systems. Additionally, stimulated processes offer a way to control surface composition and permit selective bond breaking, options which do not exist with thermal or vibrationally-stimulated means. In this article, we explore the relevance of Auger decay and Auger spectroscopy to stimulated processes.

Recently, laser resonance-enhanced multi-photon ionization spectroscopy (REMPI) has made possible the complete quantum-state characterization of neutral particles produced by the highest-probability DIET events. By this we mean that the energy distributions of the desorbing particles over the translational, rotational, and vibrational degrees of freedom are measured. The pioneering work of Burns, et al. [2] used REMPI to study electron beam stimulation of the surface. Others [3], using quantum-specific laser-inducedfluorescence (LIF) detection, have investigated laser stimulation where, although thermal desorption dominates, electronically-excited processes are also observed. The complete characterization offered by REMPI and LIF contrasts with earlier methods, such as non-selective post-ionization and massanalysis, which can measure only translational energy distributions [4], or by 
indirect means, such as observing changes in surface composition. More complete information can be found in other sources $[1,5,6]$.

Auger decay and Auger spectroscopy enter our understanding of DIET in several ways. Analysis of CVV Auger spectra allows the determination of the screened hole-hole interaction, $\mathcal{C}$, which affects both the self-trapping probability and the lifetime of two-hole excitations. Auger matrix-element effects cause deep-valence excitations in adsorbates to differ by up to several orders of magnitude in lifetime. In many systems, this means that only a few longest-lived excitations dominate the DIET yield, greatly simplifying the analysis. In separate sections, we discuss the effects of a finite $U$ and the spread in Auger rates.

\section{THE EFFECT OF BOLE-HOLE INTERACTION ON EXCITATION LIFETIME}

Consider adsorbate excitations on metallic surfaces. From photoemission spectra, one knows the single-hole valence excitation energies, $I_{j}$. From $C V$ Auger spectra, one may in principle extract the screened hole-hole interaction energy, U, using

$$
A_{j k}=I_{j}+I_{k}+U_{j k}
$$

where $A_{j k}$ are the energies of Auger peaks relative to the Fermi level. It is important to note that these are quasiparticle excitations; i.e., the screening response of the substrate (including any charge-transfer onto the adsorbate) and the relaxation energy of the adsorbate electrons are included in $I$ and A. However, one factor inherent in Eq. (1) needs to be discussed: 
Several workers have stressed the importance of mixing between two-hole configurations in understanding molecular Auger spectra, both with respect to the peak energies $[7,8]$ and inir amplitudes [9]. The reason for this is obvious: the spectral density of two-hole configurations is naturally greater than that of one-hole configurations, and when two configurations of the same symmetry are proximate in energy, they necessarily mix; the extent of mixing is determined by the interaction energy divided by the difference in configuration energies. Since Eq. (1) is based on an interacting single-particle model, configuration interaction (CI) mixing, which destroys the single-particle description of the holes, is ignored. Therefore, Eq. 1 unavoidably includes energy shifts due to CI mixing in the value of what otherwise is thought of as a screened hole-hole interaction energy, i.e., U. This complication consequently decreases the usefulness of Eq. 1 in applications to gas phase molecules; for example, different U's can be found in excitations whose electrostatics are the same. However, CI mixing can sometimes be neglected for qualitative evaluation of gas-phase spectra, either because in small molecules symmetry often prevents mixing (i.e., no two-hole configurations of the same symmetry are close in energy) or because in larger molecules a dense two-hole spectrum allows CI effects to be in some sense averaged out (see the examples in Ref. 10).

In the adsorbate case, a remarkable factor reduces the need to directly include $C I: U_{i j}$ determines the degree of correlation between quasiparticles $i$ and $j$, and the system screens this interaction, usually quite well. This is an empirical observation: comparing $U$ in the $L_{2,3} \mathrm{M}_{4,5} \mathrm{M}_{4,5}$ spectra of $\mathrm{Cu}$ [11] , $\mathrm{Ni}$ [12], and $\mathrm{Co}_{0}[13]$ metals, we find a rapid reduction from $\sim 8$, to $\sim 2$, to 0-1 eV as the $3 d$ atomic shell opens to permit charge-transfer screening into 
the same principal shell as contains the final-state holes. Additionally, even in the relatively weakly chemisorbed $C 0$ :metal systems, $U$ in $C O$ has been determined by several groups [14] to range only from 0-2 eV, despite a value of $\sim 15 \mathrm{eV}$ in the gas phase [15]. Here again, this dramatic reduction correlates with charge-transfer into the molecular $2 \pi$ level which effectively screens intramolecular holes. We do not believe any detailed understanding of this phenomenon exists: the quasiparticle structure and interactions involve many-body dynamics on the $10^{-16}-10^{-17}$ sec timescale; apparently nature is capable of almost full quasiparticle screening unless some direct impediment exists, such as closing a principal atomic shell which shuts down chargetransfer screening, as in the above $\mathrm{Co}-\mathrm{Ni}-\mathrm{Cu}$ case. This principle also explains the observation of significant U's in ionic materials, such as the high-Tc cuprate superconductors [16], and greatly reduced U's in covalent materials, in spite of significant bandgaps.

Before we discuss the impact of finite U's on DIET, we must review the basic physics of these processes. A typical energy level diagram is shown in Fig. 1, here for the $\mathrm{NO}_{2}:$ Pt system, discussed in detail in Refs. [17] and [18]. The width of the substrate valence bands is shown on the left and levels from the molecular photoemission spectrum, labeled with the principal molecular orbital of the excitation, are found on the right. Shallow single particle excitations are degenerate with substrate bands and can decay by resonant tunneling (i.e., the excitation is transferred to the substrate). Deeper valence excitations have no such degeneracy and Auger decay preferentially using electrons in shallower adsorbate orbitals (optical decay is slower than Auger; in the case of a metallic substrate, Auger decay is always possible as an arbitrary energy can be imparted to an electron at the Fermi 
leve1). Because intra-atomic Auger matrix elements dominate over interatomic ones, these decays will use electrons on the adsorbate rather than metal electrons directly. In the following section, we will examine the Auger matrix elements for these decays and find that variations in lifetime of up to two orders of magnitude are possible.

While all excitations have a finite probability of producing a DIET event, the excitations which dominate these processes, i.e. those which determine how the surface is modified, are the longest lived: Basic dynamical considerations suggest that the yield of a particular excitation, $Y$, is exponential with lifetime, or $Y \sim \exp \left(-\Gamma \tau_{c}\right)$, where $\Gamma$ is the inverse excitation lifetime and $\tau_{c}$ is the time it takes the system in the excited state to relax sufficiently that decay to the ground state still yields a product [19]. This expression breaks down if $\tau_{c} \ll<1 / \Gamma$ and becomes more complex in more than one dimension [20], but does contain the basic physics. For metallic surfaces, we expect $\tau_{c}>1 /$. Experimental evidence that this is so comes from the effects of coadsorbates. For example, Fig. 2 shows the dramatic increase in the yield of NO which occurs due to oxygen coadsorption [21]. In this system, screening charge occupies molecular $2 \pi$ orbitals which hybridize with substrate levels; $2 \pi$ occupancy directly determines the Auger decay rate of a 50 hole, which is the highest probability desorption channel. Electronegative coadsorbates reduce the $2 \pi$ screening charge density by both direct through-space electrostatic interactions and an increase in the workfunction. This dramatic rise in yield would not result from a lifetime change if $\tau_{c} \ll 1 / \Gamma$; explanations based on factors other than excitation lifetime have been ruled out [21].

Because of the strong dependence of yield on lifetime, we expect shallow valence excitations, which decay by resonant tunneling to result in yields 
which are greatly reduced compared with excitations which must Auger decay. This is because the tunneling rate $\Gamma \sim t$, the transfer integral between the relevant orbital and the substrate, and for small adsorbates $t$ is generally a few tenths of an $\mathrm{eV}$ or greater, ranging up to several eV for strongly chemisorbed systems. In contrast, a few tenths of an $\mathrm{eV}$ is an upper bound for valence Auger $\Gamma$ (see Section 4). This is consistent with the observed thresholds of the dominant processes, as seen, for example, in Fig. 3, where the observed threshold [17] for $\mathrm{NO}_{2}: \mathrm{Pt}(111)$ corresponds to the deep valence levels in Fig. 1.

One effect of finite U's in adsorbate systems may be found by considering the longest-lived two-hole excitations of $\mathrm{NO}_{2}: \mathrm{Pt}(111)$. Note that holes in shallow levels, such as the $1 a_{2}$ (Fig. 1), can be prevented from tunneling decay if $\mathrm{I}_{1 a_{2}}+\mathrm{U}>W^{\prime}$, where $W^{\prime}$ is the occupied substrate bandwidth. For the $1 a_{2}$ of adsorbed $\mathrm{NO}_{2}$, this only requires that $\mathrm{U} \geq 2-3 \mathrm{eV}$, which is reasonable. Evidence that this occurs comes from the analysis of the vibrationally- and rotationally-resolved translational energies of the desorbed dissociation fragment (NO). A bimodal structure at intermediate rotational energies suggests two channels of comparable yield, both with thresholds of $\sim 10-13 \mathrm{eV}$. On the basis of a lifetime analysis, these have been assigned to a one- $\left(3 b_{2}^{-1}\right)$ and a two-hole $\left(1 \mathrm{a}_{2}^{-2}\right)$ excitation, the latter prevented from resonant-tunneling decay by a finite $U$ [17].

\section{THE EFFECTS OF U ON EXCITATION SELF-TRAPPING}

In the case of an isolated adsorbate, an excitation which is not degenerate with the substrate valence bands is inherently self-trapped; i.e., it cannot delocalize into the metal but remains localized until it decays. In 
real systems, adsorbate coverage determines the dispersion of excitations within the adsorbate layer. As dispersion increases, there is increasing probability that a surface excitation will not self trap. This effect is visible in the coverage dependence of the yield, discussed below.

We consider in Fig. 4 the coverage dependence of the NO ESD cross section [22], where we show two channels separately. The "low energy" channel with a threshold of $\sim 10 \mathrm{eV}$ has been assigned to the $5 \sigma^{-1}$ excitation and shows pronounced changes in cross-section with coverage. The "high energy" channel with a threshold of $\sim 18 \mathrm{eV}$ has been assigned to the $50^{-2}$ excitation and shows no changes in cross section. This difference is due to the hole-hole interaction: a $U$ greater than the $5 \sigma$ bandwidth ( $\sim 1 / 2 \mathrm{eV})$ prevents the two holes from hopping apart, as this is energetically forbidden. Hopping together, the $2 \mathrm{~h}$ bandwidth is then very narrow so that self-trapping occurs with almost unit probability, regardless of coverage.

The exponential drop in cross section of the low energy channel can be attributed to the increasing dispersion of the $5 \sigma$ excitation with coverage, approaching a $5 \sigma$ bandwidth of $\sim 0.5 \mathrm{eV}$ at saturation, as revealed by photoemission. This means that competition occurs between delocalization and the self-trapping of excitations. If we assume that all excitations are inherently self-trapped at very low coverage, we see at high coverage that about $5 / 6$ of the 50 excitations delocalize. (The basic physics concerning selftrapping may be found summarized in a recent proceedings [23].) The singlehole case has been understood based on a tight-binding model [22] and the resulting curve is also shown in Fig. 4. It was found that the self-trapping probability scales as $1-\exp (-\alpha / n)$, where $n$ is the number of nearest neighbors, assumed randomly distributed for a given coverage in the model, and $\alpha=$ 
$\Delta \mathrm{E}^{2} / \beta^{2}$, where $\Delta \mathrm{E}$ is the spread in adsorbate potential energy in the excited state and $\beta$ is the adsorbate-adsorbate transfer integral. In a non-orthogonal atomic orbital representatio:., the largest term which causes two-holes to hop together is $\beta S$, where $S$ is the overlap integral between neighboring adsorbates (the Coulomb term is of order $S^{2}$ ). Since $S\langle<0.1$, with $U\rangle W$ self-trapping is assured.

\section{LIFETINE OF ADSORBATE EXCITATIONS TO VALENCE AUGER DECAY}

We have summarized the importance of lifetime arguments in finding highprobability DIET channels. We now present some details of the Auger decay process of an adsorbate valence hole. Let us assume that realistic MOs are available from cluster calculations for the adsorbate-substrate complex, where these are local representations of the extended orbitals. Because the screening relaxation time is an order of magnitude shorter than the Auger lifetime, only the completely relaxed valence-hole state need be considered (nonorthogonality of the MOs of the fully-relaxed Auger initial state and those of the Auger final state leads to shake-up and shake-off phenomena [10], which are of consequence to spectroscopy but not to the present discussion).

The Auger amplitude is proportional to two-electron matrix elements of the form $\iint d\left(r_{1}\right) d\left(r_{2}\right) \phi_{i}^{*}\left(r_{1}\right) \phi_{j}\left(r_{1}\right) e^{2} / r_{12} \phi_{k}^{*}\left(r_{2}\right) \phi_{o}\left(r_{2}\right)$. Here an initial-state valence hole in MO $\phi_{i}$ decays through the use of valence electrons in MOs $\phi_{j}$ and $\phi_{k}$ resulting in an outgoing electron in continuum orbital $\phi_{0}$. A very important point, argued on theoretical grounds and by comparison to molecular Auger experiments [10], is that the MOs $\phi_{j}$ and $\phi_{k}$ in the matrix element are initial state orbitals. This means that charge-transfer screening directly affects the Auger rates if $\phi_{j}$ and/or $\phi_{k}$ are adsorbate-metal hybrids. A second 
important point is that intra-atomic matrix elements are an order of magnitude or more larger than inter-atomic matrix elements. This means that if we expand the MOs in atomic orbitals, we may estimate the Auger rate by summing only single site terms. If we are interested in relative rates rather than absolute rates, this approximation is even better.

The dominance of intra-atomic terms means that if an adsorbate hole uses electrons in a metal-adsorbate hybrid orbital for decay (such as a $5 \sigma$ hole decaying with $2 \pi$ electrons in $N(P t)$, the decay rate scales as the square of the amplitude of the adsorbate component of the hybrid orbital. (Channels which directly use metal orbitals are much smaller.) Recently, an empirical CI model has suggested that the giant yield enhancement seen in Fig. 2 could indeed be explained by workfunction-induced changes in this hybridization [21].

Another factor which determines the Auger lifetime is the angular momentum transfer between the electrons. Expanding the MOs in atomic orbitals (AOs) and neglecting to first order the interatomic contributions, angular momentum transfer appears as a factor of $1 /(2 \ell+1)$ in the $A 0$ matrix elements, a result of the expansion of $e^{2} / r_{12}$ in spherical coordinates. This transfer is $\ell=0$, 1 or 2 for decays involving the $2 \mathrm{~s}$ and $2 p$ shells, corresponding to Auger-rate factors of 1 for intra-orbital decays, $1 / 9$ for $s-p$ decays, and $1 / 25$ for px-py, px-pz, or py-pz decays. Calculations using the actual orbitals determine the relative weights and include the charge density explicitly. The longest-lived excitations have lifetime widths $\Gamma$ approaching $0.01 \mathrm{eV}$ in $N 0$, for example $[2,24]$.

The above factors lead to a simple rule for finding the longest-lived valence excitations: these are holes not degenerate with occupied substrate 
bands which must be filled by electrons from different A0s. For example, in the case of NO or $\mathrm{CO}$, the $5 \sigma^{-1}, 5 \sigma^{-2}$, and $5 \sigma^{-2} 4 \sigma^{-1}$ excitations should be longlived because these orbitals are hybrids of $2 \mathrm{~s}$ and $2 \mathrm{pz} \mathrm{AOs}$, and they decay using $2 \pi$ electrons which are hybrids of $2 \mathrm{px}$ and $2 \mathrm{py} \mathrm{A} 0 \mathrm{~s}$. In contrast, the electron density in the $2 \pi$ and $5 \sigma$ orbitals make $1 \pi$ and $4 \sigma$ holes short lived, respectively. As symmetry is reduced, this rule is less useful because the molecular orbitals are more complex mixtures of different atomic orbitals.

Recently, coincidence Auger experiments by Bartynski and coworkers [25] have shown that bulk density-of-states (DOS) features can be seen in the spectra of core holes produced in surface atoms. While some have suggested that this could be explained by a low-energy Auger decay which moves the core hole to a bulk atom, another question is exactly how local is the DOS probed by an Auger process? This in turn questions the adequacy of the single-site approximation employed above. Naturally, the single-site question depends entirely on the representation of the valence functions, i.e., LCAO vs. muffintin orbitals, for example. Long ago [26], numerical tests of true interatomic matrix elements in an LCAO representation showed them to be negligible in a covalent material, $\mathrm{Si}$. However, in Fig. 5 we show recent results by Jennison and Weightman [27] who found that for low-energy decays, the intra-atomic matrix elements are slowly converging with $R$, with considerable current supplied outside what would be a muffin-tin radius of 2.2 a.u. (This dependence was found by integrating out the first radial coordinate in the above expression, the one involving the product of the (core) hole and valence wavefunctions, and plotting the resulting one-dimensional function whose integral is the matrix element itself.) This result opens the possibility that nearest-neighbor DOS features could affect the Auger spectra, even though the 
single-site approximation is sound in a LCAO formalism. However, the above arguments concerning adsorbate excitation lifetime differences still hold, because they are due to simple symmetry arguments and arise from the angular part of the matrix element.

\section{ACKNOWLEDGEYENTS}

This work was performed at Sandia National Laboratories, supported by the US Department of Energy under contract DE-AC04-76DP00789.

\section{REFERENCES}

1. Desorption Induced by Electronic Transitions: a) DIET I, N. H. Tolk, M. M. Traum, J. C. Tully, and T. E. Madey, eds., Springer-Verlag (1.983);

b) DIET II, W. Brenig and D. Menzel, eds., Springer-Verlag (1985);

c) DIET III, R. H. Stulen and M. L. Knotek, eds., Springer-Verlag (1988);

d) DIET IV, G. Betz and P. Varga, eds., Springer-Verlag (1990).

2. A. R. Burns, E. B. Stechel and D. R. Jennison, Phys. Rev. Lett. 58, 250 (1987); in Ref. 1c, p. 67.

3. S. A. Buntin, L. J. Richter, R. P. Cavanagh and D. S. King, Phys. Rev. Lett. 61, 1321 (1988).

4. P. Feulner, et al., in Ref. 3, p. 58.

5. D. R. Jennison, A. R. Burns, and E. B. Stechel, Rev. Solid State Sci. $\underline{4}$, 699 (1990), and references therein.

6. P. Avouris and R. E. Walkup, Annu. Rev. Phys. Chem. 40, 173 (1989).

7. C.-M. Liegener, Chem. Phys. 92, 97 (1985).

8. P. Weightman, T. D. Thomas and D. R. Jennison, J. Chem. Phys. $\underline{78}, 1652$ (1983).

9. D. R. Jennison, Chem. Phys. Letts. 69, 435 (1980).

10. D. R. Jennison, Phys. Rev. A 23, 1215 (1981).

11. S. P. Kowalczyk, et al., Phys. Rev. B $\underline{8}, 2387$ (1973).

12. T. Jach and C. J. Powell, Phys. Rev. Lett. 40, 964 (1978). 
13. D. Y. Zehner, J. R. Noonan and H. H. Madden, J. Vac. Sci. Technol. 20, 859 ( $९$ \$2); D. K. G. de Boer, C. Haas and G. A. Sawatzky, J. Phys. F: Met. Phys. 14, 2769 (1984).

14. B. E. Koel, J. M. White, and G. M. Loubriel, J. Chem. Phys. 77, 2665 (1982); E. Umbach and Z. Hussain, Phys. Rev. Lett. 52, 257 (1984).

15. D. R. Jennison, et al., Phys. Rev. Lett. 46, 913 (1981); C. W. Bauschlicher and P. S. Bagus, J. Chem. Phys. 80, 944 (1984).

16. E. B. Stechel and D. R. Jennison, Phys. Rev. B $\underline{38}, 4632$ (1988) and references therein.

17. A. R. Burns, E. B. Stechel and D. R. Jennison, Phys. Rev. B $\underline{40}, 9485$ (1989) .

18. T. M. Orlando, A. R. Burns, E. B. Stechel and D. R. Jennison. Phys. Rev. B, submitted; J. Vac. Sci. Tectiol. A $\underline{9}, 1769$ and 1774 (1991).

19. D. Menzel and R. Gomer, J. Chem. Phys. 41, 3311 (1964); P. E. Redhead, Can. J. Phys. 42, 886 (1964); for a more recent review, see R. Gomer, in Ref. 1a, p. 40.

20. E. B. Stechel, in Ref. 1c, p. 136.

21. A. R. Burns, et al., Phys. Rev. B, in pass; J. Vac. Sci. Technol. A $\underline{8}$, 2705 (1990); Ref. 1d, p. 182.

22. A. R. Burns, D. R. Jennison and E. B. Stechel, J. Vac. Sci. Technol. A 5, 671 (1987).

23. Y. Toyozawa, Rev. Sol. St. Sci. $\underline{4}, 133$ (1990).

24. D. R. Jennison, in Ref. 1c, p. 167.

25. E. Jensen, et al., Phys. Rev. B $\underline{41}, 12468$ (1990).

26. D. R. Jerniscn, Phys. Rev. Lett. 40,807 (1978).

27. D. R. Jennison and P. Weightman, J. Vac. Sci. Technol., submitted.

\section{DISCLAIMER}

This report was prepared as an account of work sponsored by an agency of the United States Government. Neither the United States Government nor any agency thereof, nor any of their employees, makes any warranty, express or implied, or assumes any legal liability or responsibility for the accuracy, completeness, or usefulness of any information, apparatus, product, or process disclosed, or represents that its use would not infringe privately owned rights. Reference herein to any specific commercial product, process, or service by trade name, trademark, manufacturer, or otherwise does not necessarily constitute or imply its endorsement, recommendation, or favoring by the United States Government or any agency thereof. The views and opinions of authors ; xpressed herein do not necessarily state or reflect those of the United States Gnvernment ot any agency thereor. 


\section{FIGURE CAPTIONS}

1. Energy Levels of $\mathrm{NO}_{2}: \mathrm{Pt}(111)$.

2. Yield of NO vs. Coverage of Coadsorbed Oxygen from N0:Pt(111).

3. Thresholds for the Dominant Stimulated Dissociation Processes from $\mathrm{NO}_{2}: \mathrm{Pt}(111)$.

4. The Coverage Dependence of the NO Yield from NO:Pt(111).

5. The Radial Dependence of the $\mathrm{L}_{23}$ VV Natrix Elements of Si. The solid, dashed, and dotted lines indicate $s^{-}, p_{-}$, and d-wave elements, respectively. 


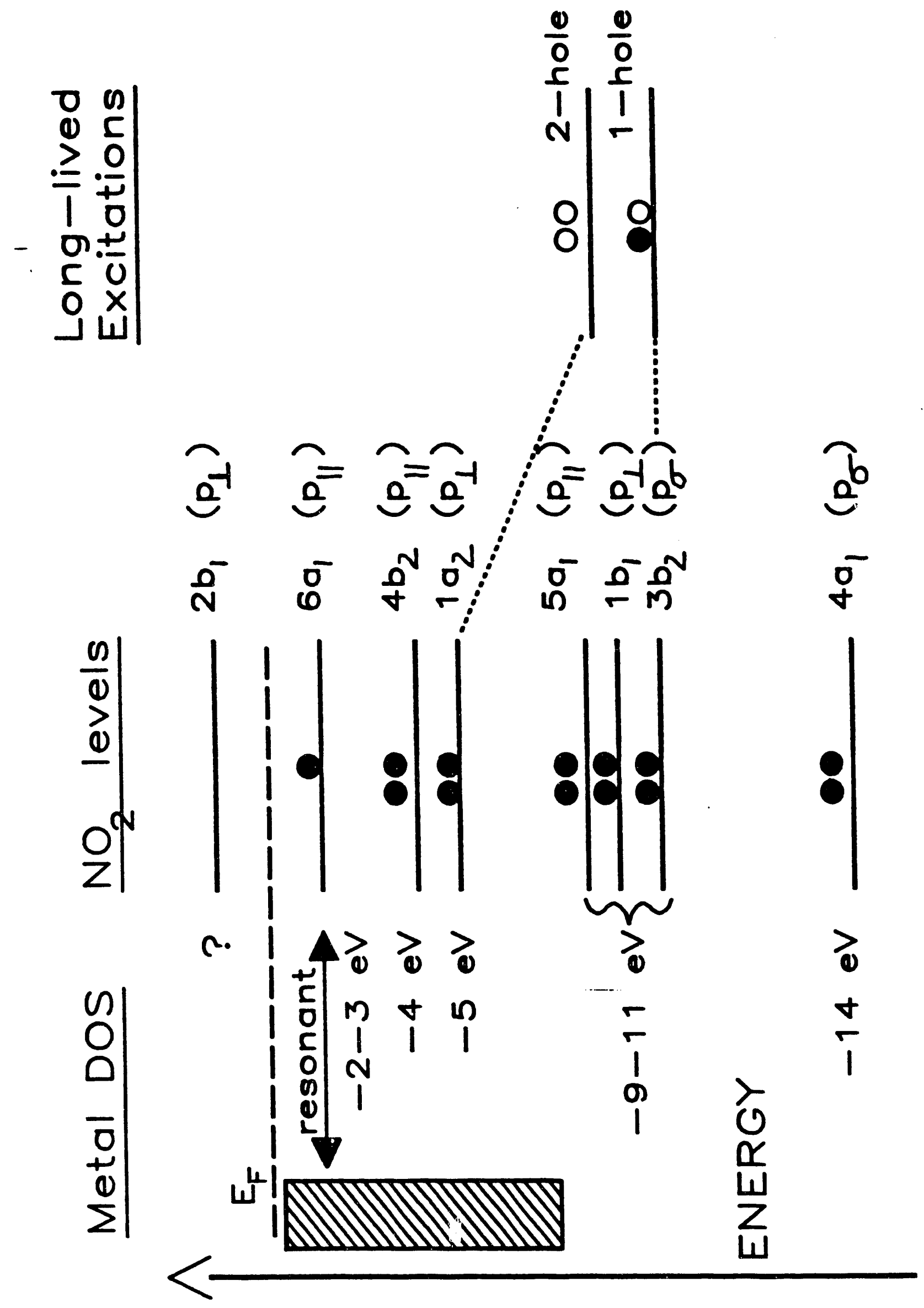




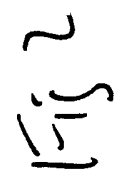

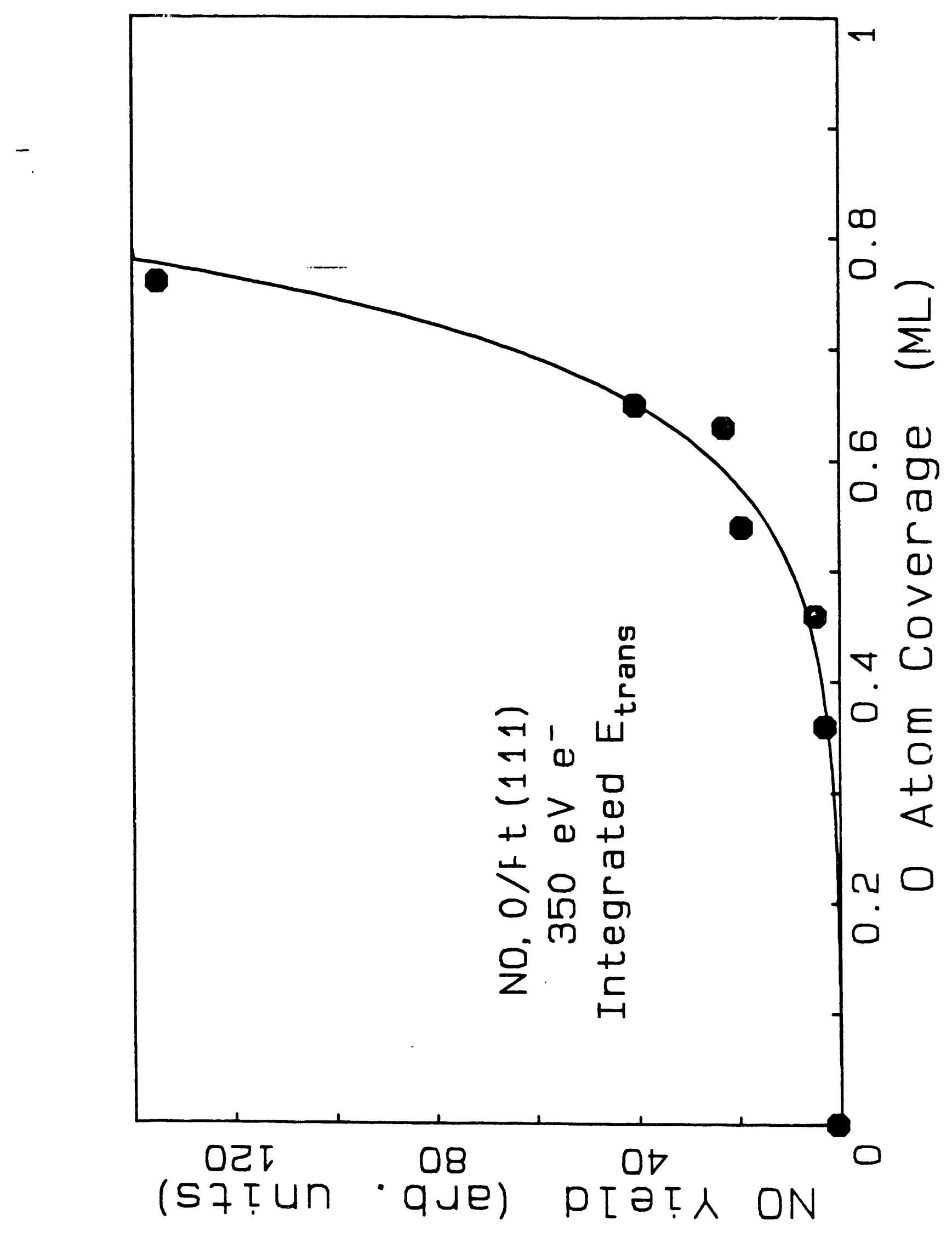


i

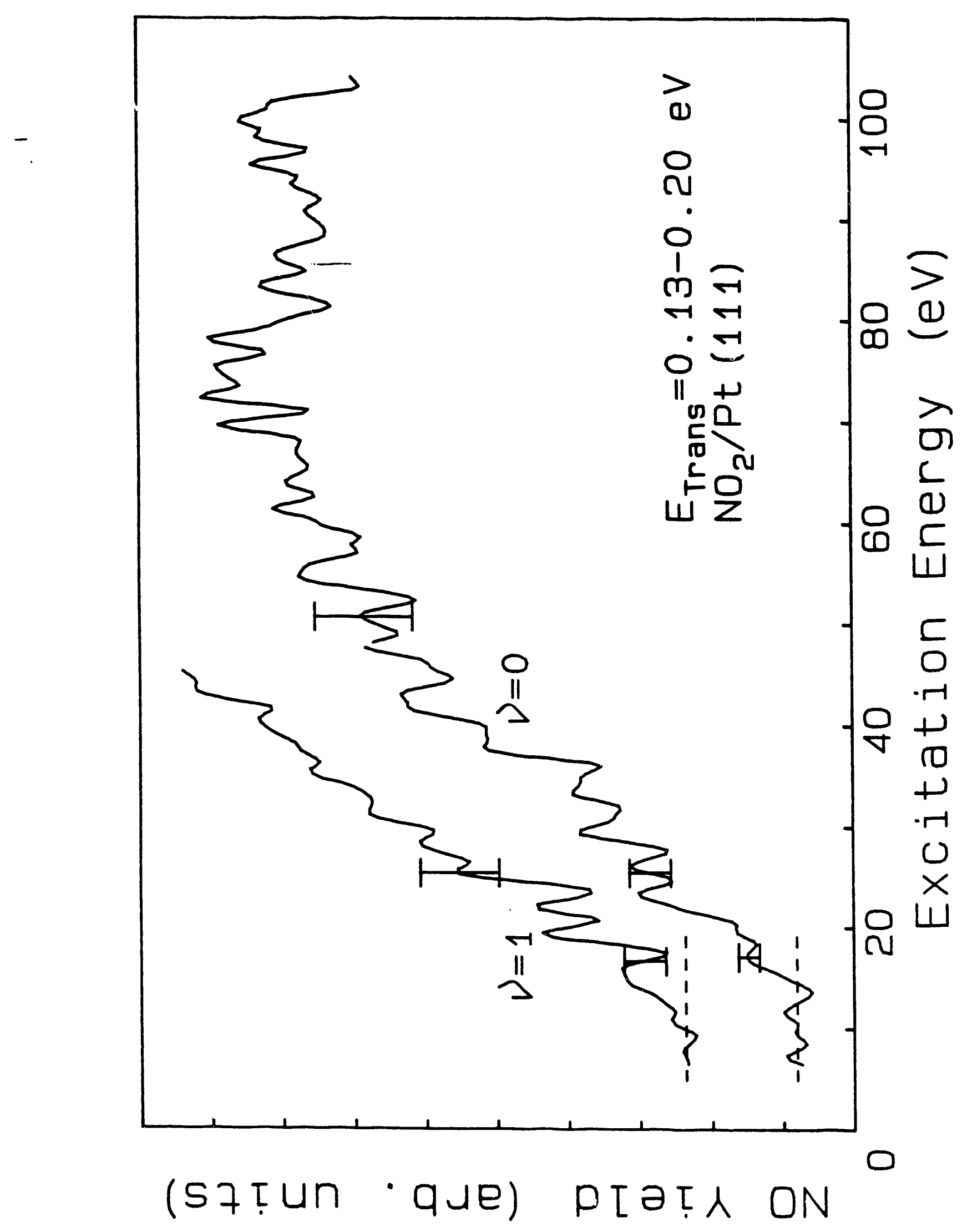



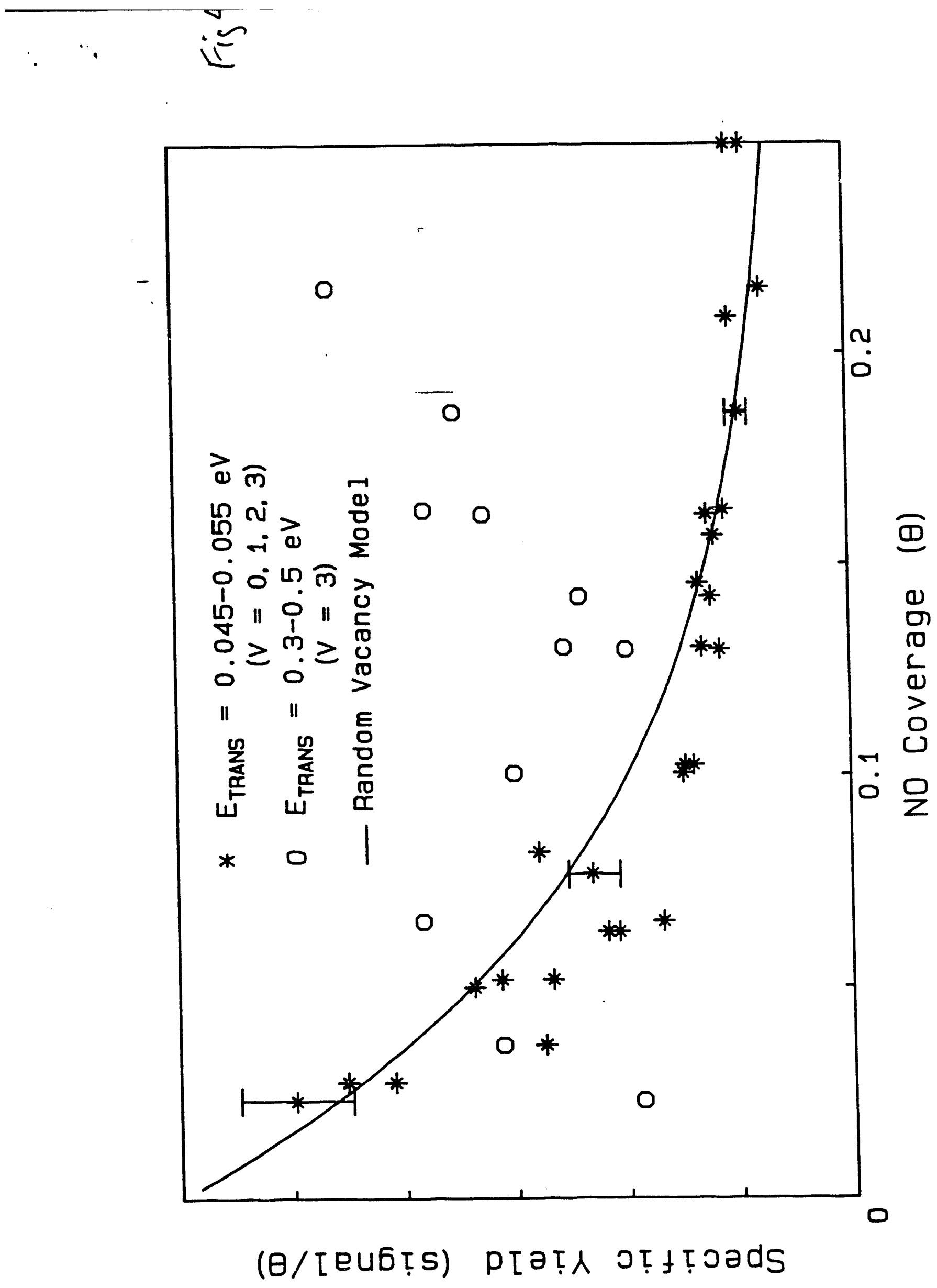


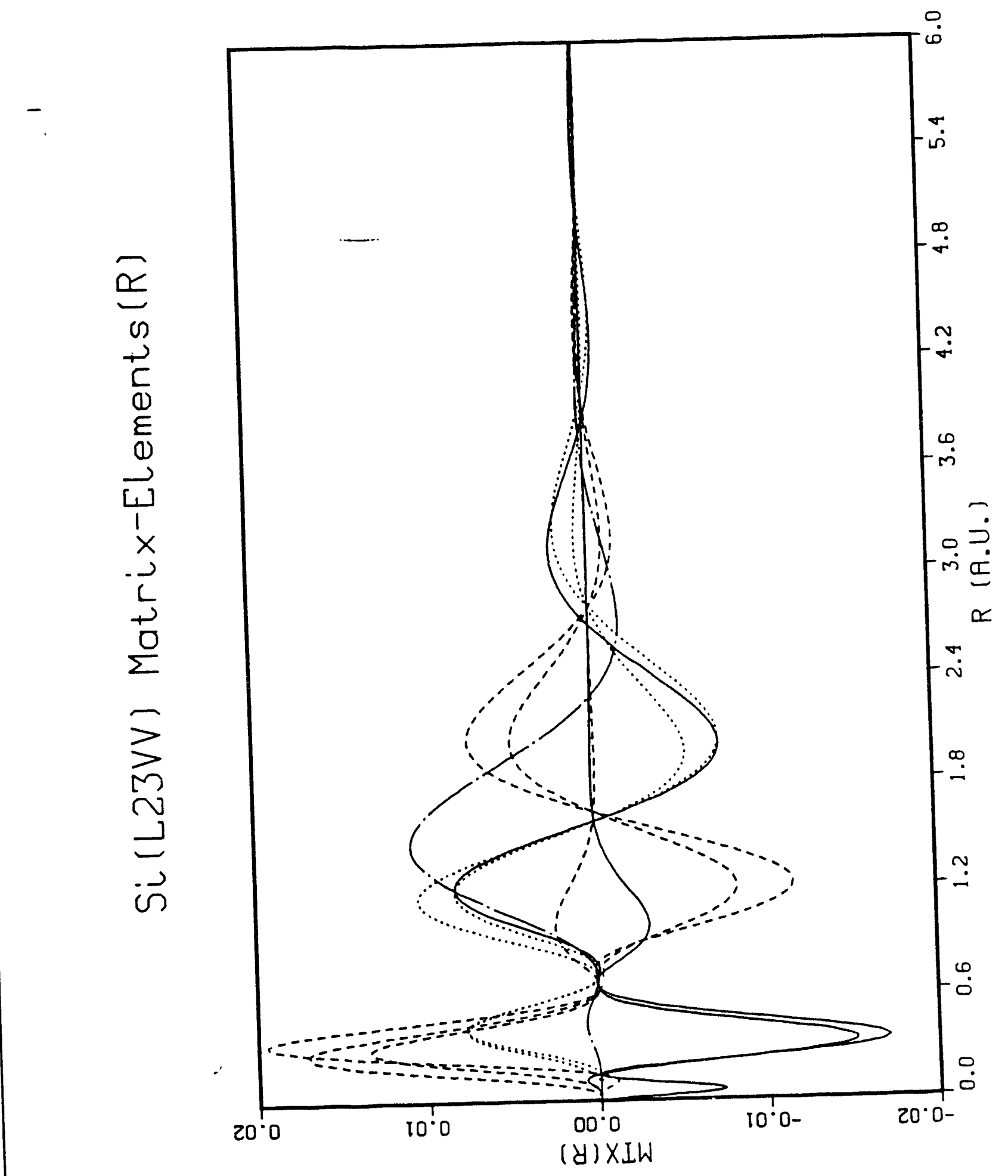



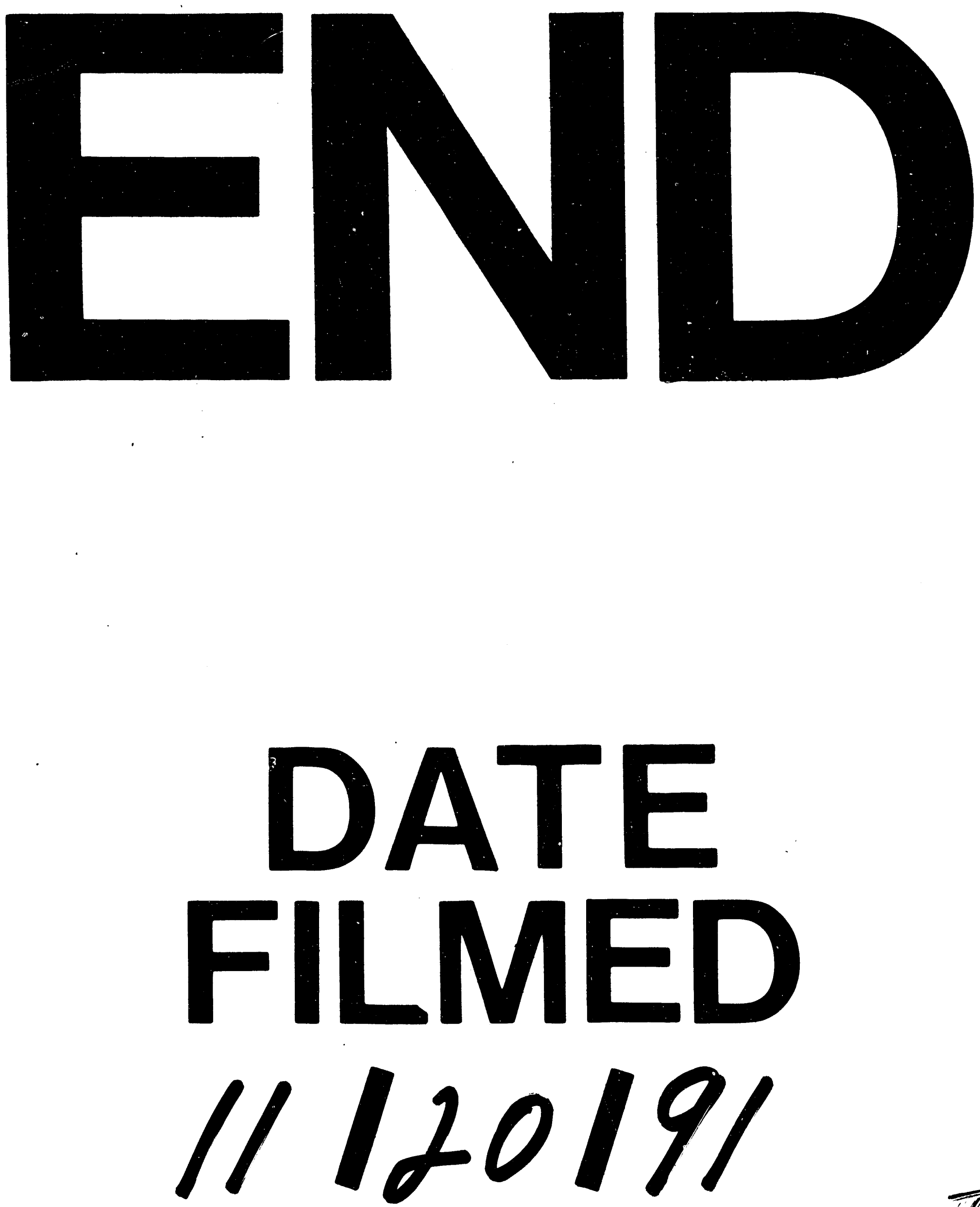
